\title{
On the effects from the simultaneous occurrence of the critical Casimir and dispersion forces between conical colloid particle and a thick plate immersed in nonpolar critical fluid
}

\author{
Galin Valchev ${ }^{1, \star}$ \\ ${ }^{1}$ Institute of Mechanics, Bulgarian Academy of Sciences, Acad. G. Bonchev St., Block 4, 1113 Sofia, Bul- \\ garia.
}

\begin{abstract}
Here we study the interplay between the van der Waals (vdWF) and critical Casimir forces (CCF), as well as the total force (TF) between a conical colloid particle and a thick planar slab. We do that using general scaling arguments and mean-field type calculations utilizing the so-called "surface integration approach", a generalization of the well known Derjaguin approximation. Its usage in the present research, requires knowledge on the forces between two parallel slabs, confining in between some fluctuating fluid medium characterized by its temperature $T$ and chemical potential $\mu$. The surfaces of the colloid particle and the slab are assumed coated by thin layers exerting strong preference to the liquid phase of a simple fluid, or one of the components of a binary mixture, modeled by strong adsorbing local surface potentials, ensuring the so-called $(+,+)$ boundary conditions. On the other hand, the core region of the slab and the particle, influence the fluid by long-ranged competing dispersion potentials. We demonstrate that for a suitable set of colloid-fluid, slab-fluid, and fluid-fluid coupling parameters the competition between the effects due to the coatings and the core regions of the objects, result, when one changes $T$ or $\mu$, in sign change of the Casimir force (CF) and the TF acting between the colloid and the slab. Such an effect can provide a strategy for solving problems with handling, feeding, trapping and fixing of microparts in nanotechnology.
\end{abstract}

\section{Introduction}

In a recently published work [1], it was shown that the total force (TF) between a spherical colloidal particle and a thick planar substrate, as well as between two spherical particles immersed in some critical nonpolar medium, exhibits not only quantitative, but also qualitative control by simple change of some external to the system parameters like the temperature $T$ and the chemical potential $\mu$ of the fluid or the minimal separation $L$ between the interacting objects. This study was based on the findings in an earlier research [2], related to the emergence and interplay between the CCF and dispersion $\mathrm{vdWF}$ within the gap formed by couple of parallel planar plates, confining a nonpolar fluid which is thought to be at the vicinity of its bulk critical point. There it was shown that the supposed interplay leads to sign change of the net interaction, due to variation of any of the aforementioned external parameters, when the film thickness is less than some threshold value $L_{\text {crit }}$.

^e-mail: gvalchev@imbm.bas.bg 
In the present article we are going to be interested in the simultaneous manifestation of both the $\mathrm{CCF}$ and the vdWF, which gives rise to the total force $F_{\text {tot }}$ between a tip like object, say the ending of a cantilever of an atomic force microscope, and a thick smooth plate. In particular, we will resemble such an object with a conical in shape colloidal particle with mesoscopic radius $R$ and height $H$, immersed in a medium that is either simple fluid or a binary liquid mixture. The mutual orientations which shall be considered include: base and/or apex of the cone facing the plate, together with the case when the height of the cone is parallel to the plane bounding the plate. In all three geometries the minimal surface-to-surface approach between the objects will be designated by $L$. Furthermore we shall envisage that the fluid is in the vicinity of its bulk critical point, with the critical temperature $T_{c}$ interpreted either as that at the liquid-vapor critical point of a simple fluid or as that at the critical demixing point of a binary liquid mixture. When such conditions are present, as first suggested by Fisher and de Gennes [3], an additional component adds up to the already acting forces if any, resulting from the critical fluctuation of the medium. This new contribution is of a long-ranged character and strongly depends on the boundary conditions which the bodies immersed in the medium impose on it at their surfaces. Since this fluctuation-induced force (FIF) depends additionally only on some gross features of the fluid medium $[4,5]$ it can be treated as universal in nature. One can make a parallel with the force between neutral bodies due to the quantum and temperature fluctuations of the charge distributions in them, i.e., of the electromagnetic field, which force is known today under the general name of quantum electrodynamic (QED) Casimir force [6-8]. Because of this analogy it became customary to term the fluctuation part $F_{\mathrm{Cas}}$ of the total force near $T_{c}$ critical Casimir force $[4,5]$.

In a medium where both quantum and thermodynamical fluctuations are present, $F_{\text {tot }}$ can be thought as a sum of a regular background $F_{\text {tot }}^{(\mathrm{reg})}$ and a singular $F_{\text {tot }}^{(\mathrm{sing})}$ contributions. The first depends in an analytic way on the parameters characterizing the medium, whereas the latter emerges due to the critical fluctuations of the medium, respectively. Therefore

$$
F_{\mathrm{tot}}=F_{\mathrm{tot}}^{(\mathrm{reg})}+F_{\mathrm{tot}}^{(\mathrm{sing})}
$$

Undoubtedly, if a system is composed out of objects, where at least one has nonplanar geometry, its excess grand canonical potential $\Omega_{\mathrm{ex}}(T, \mu \mid L, \cdots)$, or excess free energy, will show dependance on some specific geometrical characteristics like $L, R$ and $H$ in the case considered here. Thus we have

$$
F_{\mathrm{tot}}=-\frac{\partial}{\partial L} \Omega_{\mathrm{ex}}(L \mid T, \mu, \cdots) \text {. }
$$

It is normal to equate

$$
F_{\text {Cas }} \equiv F_{\text {tot }}^{(\text {sing })}(L \mid T, \mu) \text { and } F_{\mathrm{vdW}} \equiv F_{\text {tot }}^{(\text {reg })}(L \mid T, \mu),
$$

since the dispersion van der Waals interactions, ubiquitous for any system, are not influenced by the thermodynamic fluctuations.

In the past few decades the rapid development of the experimental techniques led to the emergence of the field of nanotechnology, where fluctuation-induced phenomena, bearing in mind the vdWF and QED CF, play a dominant role between neutral nonmagnetic objects when placed at distances less than a micrometer. The general theory of the FIFs with quantum character together with the subsequent experiments for its validation, have shown undeniably that these forces lead to irreversible, usually undesirable phenomena, such as stiction (i.e., irreversible adhesion) or pull-in due to mechanical instabilities [9-11] when the interactions take place in vacuum or gas medium. Moreover the weight of a particle becomes negligible when its size is scaled below a micrometer. As a result, when one tries to release such a neutral particle from, say, the surface of whatever handling device in air or 
vacuum, the particle will not drop down under the gravity but, instead, will stick to the surface due to the effect of the omnipresent vdWF. In that respect it seems adequate to introduce some fluid medium, which properties can lead to modification of the net interaction between the manipulated particle and the operating device, sometimes called gripper, in such a way that it is repulsive at short distances between the handling surfaces and the particle and attractive at larger ones. In the light of a potential experimental realization the choice of a chemically inert fluid medium absent of free changes dissolved in it seems suitable, since from one side any complications with isolation of all possible circuitry at small distances will be minimized, and on the other no chemical reactions with the substances of the particles will take place. That leads us to choose as a fluid a nonpolar liquefied noble gas that has critical parameters as close as possible to the normal ones.

In the current article we will demonstrate that by proper choice of the materials (cores) of the colloid particles and the handling surface of a gripper it is indeed possible to achieve control over the net interaction (TF) as well as the CCF between the surface and the particle by simply changing $T$ and $\mu$. We must note that so far the behaviour of the CCF has never been studied in the geometry under consideration here, namely the interaction between a cone and a plate. Let us also stress that due to its unique temperature dependence, the CCF allows in situ control of reversible assembly in soft matter and nanoscience. A further advantage of the force is that both its magnitude and range of action depends on the separation between the objects and the thermodynamic parameters of the fluctuating medium. The last facts can potentially be used in controlling the properties of colloidal suspensions and for governing the behavior of objects at small, below micrometer, distances.

The content of the article is arranged as follows. In Subsec. 2.1 we present some basic results of the finite-size scaling theory. In Subsec. 2.2 we recall and comment on the finite-size behaviour of systems with dispersion forces extending the known facts to the expected behaviour of the CCF, vdWF and TF when they act between pair of parallel plates. By doing so, we introduce the general technique, namely the "surface integration approach" (SIA) (Subsec. 2.3), within which we study the commented spectrum of forces. Section 3 briefly presents the corresponding lattice-gas model suitable for the investigation of fluid media with account of the long-ranged van der Waals interactions. Here we present the equation for the equilibrium profile of the finite-size order parameter, identify the main coupling parameters characterizing the interactions in the systems, which enter in it, and give the general expressions used to calculate the CCF and TF. The article ends with Sec. 4, where we present the exact equations (Subsec. 4.1) used to obtain the numerical results for the behaviour of the investigated forces, and comment them in details in Subsec. 4.2.

\section{Theoretical background}

\subsection{General predictions of the finite-size scaling theory}

If an object of certain shape is introduced in a medium, it alters its thermodynamic behaviour in such a way that any quantitative occurrence shows dependance on the penetration depth of the symmetry breaking effect into the volume. The range to which this effect is felt within the system depends both on the scope of the interactions and on that of the correlations between the fluctuations in the fluid, which mediate the interactions between the bodies. The range of the correlations is set by the correlation length $\xi$ of the order parameter of the medium. This quantity becomes large, and theoretically diverges, at the vicinity of the bulk critical point $\left(T_{c}, \mu_{c}\right): \xi\left(T \rightarrow T_{c}^{+}, \mu=\mu_{c}\right) \simeq \xi_{0}^{+} t^{-v}$, where $t=\left(T-T_{c}\right) / T_{c}$, and $\xi\left(T=T_{c}, \mu \rightarrow \mu_{c}\right) \simeq \xi_{0, \mu}\left|\Delta \mu /\left(k_{B} T_{c}\right)\right|^{-v / \Delta}$, where $\Delta \mu=\mu-\mu_{c}$. Here $v$ and $\Delta$ are the usual critical exponents which, for classical fluids, are those of the three-dimensional Ising model, and $\xi_{0}^{+}$and $\xi_{0, \mu}$ are the corresponding nonuniversal amplitudes of the correlation length along the $t$ and $\mu$ axes. When $\xi$ becomes comparable to the characteristic dimension of the system, 
say the separation $L$ between the objects, the size dependence of the thermodynamic functions enters into the thermodynamic potentials through the ratio $L / \xi$, and takes a scaling form given by the finitesize scaling theory [5].

In the remainder we are going to study a system governed by long-ranged dispersion interactions utilizing the recently introduced "surface integration approach" [12]. For this purpose we will need the corresponding results for a system with a film geometry that is governed by the same interactions in question. That is why we are next going to concisely recall these topics. We start with the behavior of the thermodynamic Casimir force in nonpolar fluid systems with plane parallel geometry, in which the dispersion forces are accounted for.

\subsection{Influence of the dispersion forces on the thermodynamic Casimir one in nonpolar fluid systems with film geometry}

We consider a fluid system consisting of a nonpolar medium $\mathbb{M}$ comprising two thick parallel plates of materials $\mathbb{S}_{1}$ and $\mathbb{S}_{2}$ which are coated by thin solid films of substances $\mathbb{L}_{1}$ and $\mathbb{L}_{2}$, respectively - see Fig. 1(a) in [1]. If the fluid medium is in contact with a particle reservoir with a chemical potential $\mu$, the grand canonical potential $\Omega_{\mathrm{ex}}(L \mid T, \mu)$ per unite area $\mathscr{A}$ of this medium in excess to its bulk value $\mathscr{A} L \omega_{\text {bulk }}(T, \mu)$ depends on the film thickness $L$ and, thus, one can define the total effective force $F_{\text {tot }}(L \mid T, \mu)$ [in a full accord with Eq. (2)], which is due to fluctuations of the medium and dispersion interactions in it. Here $\omega_{\text {bulk }}(T, \mu)$ is the density of the bulk grand canonical potential, and $\mathscr{A}$ is the surface area of the plates.

The contribution of the dispersion forces to the total effective force Eq. (2) can be distinguished from that of the CCF by their temperature dependence, because the leading such of the former does not exhibit a singularity. Thus, one can perform the aforementioned decompositions and identifications given in Eqs. (1) and (3).

If the system is away from its bulk critical point, for the occurring force $f_{\text {tot }}^{\|}(L \mid T, \mu)$ per cross section area $\mathscr{A}$ and $k_{B} T \equiv \beta^{-1}$ is customary to write the following expression

$$
f_{\mathrm{tot}}^{\|}(L \mid T, \mu) \simeq(\sigma-1) \beta H_{A}(T, \mu) L^{-\sigma} \vartheta^{\sigma-d},
$$

where, for dimensional reasons, the microscopic length scale $\vartheta$ is introduced. Let us note that one normally considers the case $d=\sigma$ and, thus, omits the apparent dependence of this length, that can be taken to be, e.g., the so-called retardation length $[12,13] \xi_{\text {ret. }}$ In Eq. (4) $H_{A}$ is the Hamaker term, whose dependence from the temperature and chemical potential is given by the so-called Hamaker constant $[14,15]$ (for details see the Appendix in [2]).

As already explained above, upon approaching the bulk critical point of the system the fluctuations of the order parameter of the confined fluid medium exhibit strong correlations which gives rise to new contribution to the TF, the CCF (see below). In the vicinity of this point (critical region) Eq. (4) is no longer valid. Following [2], it becomes

$$
f_{\text {tot }}^{\|}(L \mid T, \mu) \simeq L^{-d} X_{\text {crit }}^{\|}\left[x_{t}, x_{\mu}, x_{l},\left\{x_{s_{i}}, i=1,2\right\}, x_{g}\right]+(\sigma-1) \beta H_{A}(T, \mu) L^{-\sigma} \vartheta^{\sigma-d} .
$$

Here $X_{\text {crit }}^{\|}$is dimensionless, universal scaling function, $x_{t}=t\left(L / \xi_{0}^{+}\right)^{1 / v}$ and $x_{\mu}=\beta \Delta \mu\left(L / \xi_{0, \mu}\right)^{\Delta / v}$ are the temperature and field relevant in renormalization group sense scaling variables, whereas $x_{l}$, $x_{s_{i}}, i=1,2$ and $x_{g}$ are irrelevant scaling variables associated with the interactions in the system. Explicitly these variables are defined in the text below Eq. (2.6) in [2].

According to the scaling hypothesis of the CCF one expects that near the bulk critical point

$$
f_{\mathrm{Cas}}^{\|}(L \mid T, \mu)=L^{-d} X_{\mathrm{Cas}}^{\|}\left(x_{t}, x_{\mu}, \cdots\right),
$$


where $X_{\mathrm{Cas}}^{\|}$is a scaling function, that for large enough $L$ with fixed $x_{t}=\mathscr{O}(1)$ and $x_{\mu}=\mathscr{O}(1)$ approaches the scaling function of the short-ranged system $X_{\text {Cas,sr }}^{\|}\left(x_{t}, x_{\mu}\right)$ (for details see Eqs. (2.12) and (4.10) in [2]). From Eqs. (1) and (3), together with Eq. (5) follows that the scaling function of the CCF $X_{\text {Cas }}^{\|}$is proportional to the sum of $X_{\text {crit }}^{\|}$and the singular part of the Hamaker term $H_{A}^{(\operatorname{sing})}(T, \mu)$. The last implies that in order to determine the CF in systems with dispersion interaction one has to decompose the contribution captured through the Hamaker term in a singular and a regular parts, i.e.

$$
H_{A}(T, \mu)=H_{A}^{(\mathrm{reg})}(T, \mu)+H_{A}^{(\text {sing })}(T, \mu) .
$$

Thus, with $d=\sigma$ one has

$$
f_{\text {Cas }}^{\|}=L^{-d}\left[X_{\text {crit }}^{\|}+(d-1) \beta H_{A}^{(\text {sing })}\right],
$$

while $F_{\mathrm{vdW}}$ from Eq. (3), normalized per unit area, in the general case coincides with Eq. (4), with $H_{A} \equiv H_{A}^{(\text {reg })}$.

In what follows, we are going to present results for the CCF, vdWF and TF in the cases of coneplate system, utilizing the knowledge gained from studies of the corresponding interactions between parallel plates.

\subsection{The van der Waals and Casimir forces for a cone-plate geometry within the SIA}

The most common approach used to calculate geometry dependent interaction energy or force in systems where at least one of the objects has a nonplanar geometry, is that first proposed by B. Derjaguin in 1934 [16]. The Derjaguin approximation (DA) suggests that one can relate the interaction force/potential between two gently curved colloidal particles with the knowledge for that between a pair of parallel plates, provided that the separation between the colloids is much smaller than their geometrical characteristics. This restriction imposes certain limitations on the usage of the DA. The necessity of more general technique as an improvement of the DA, led to the proposal of the co-called "surface integration approach" reported in [12]. It has been used there to study van der Waals interactions between objects of arbitrary shape and a plate of arbitrary thickness. It delivers exact results if the interactions involved can be described by pair potentials. The main advantage of this approach over the DA is that one is no longer bound by the restriction that the interacting objects must be much closer to each other than their characteristic sizes. The main result is that for the force acting between a $3 \mathrm{D}$ object (say a colloid particle) $B \equiv\{(x, y, z),(x, y, z) \in B\}$ of general shape $S(x, y)=z$ and a flat surface bounded by the $(x, y)$-plane of a Cartesian coordinate system, one has

$$
F_{\mathrm{SIA}}^{B, \mid}(L)=\int_{A_{S}^{\text {to }}} \int f_{\mathscr{A}}^{\|}[\mathrm{S}(x, y)] \mathrm{d} x \mathrm{~d} y-\int_{A_{S}^{\text {away }}} \int f_{\mathscr{A}}^{\|}[\mathrm{S}(x, y)] \mathrm{d} x \mathrm{~d} y,
$$

where $A_{S}$ is the projection of the surface $S$ of the particle over the $(x, y)-$ plane, $A_{S}=A_{S}^{\text {to }} \cup A_{S}^{\text {away }}$. Equation (9) has a very simple intuitive meaning: in order to determine the force acting on the particle one has to subtract from the contributions stemming from surface regions $A_{S}^{\text {to }}$ that "face towards" the projection plane those from regions $A_{S}^{\text {away }}$ that "face away" from it, where $A_{S}^{\text {to }}$ and $A_{S}^{\text {away }}$ are the projections of the corresponding parts of the surface of the body on the $(x, y)$-plane. It is clear that if one takes into account only the contributions over $A_{S}^{\text {to }}$ one obtains expression very similar to the DA. Both expressions in that case will differ only by the fact that while Eq. (9) takes into account that the force on a given point of $S$ is along the normal to the surface at that point, the standard DA does not take this into account. Let us recall that Eq. (9) provides exact results for the interaction under the assumption that the constituents of the body interact via pair potentials. This is, of course, not the case 
of CCF. It is, however, clear that under mechanical equilibrium of the colloid in the fluid, the CCF is again along the normal to the surface at the point of the surface where it acts. Thus, one can get a reasonably good approximation to the effect of that force by keeping just the integration over the part of the surface of the body that faces the plane. This leads to

$$
\beta F_{\mathrm{Cas}, \mathrm{SIA}}^{B, \mid}(L)=\int_{A_{S}^{\mathrm{to}}} \int f_{\mathrm{Cas}}^{\|}[\mathrm{S}(x, y)] \mathrm{d} x \mathrm{~d} y .
$$
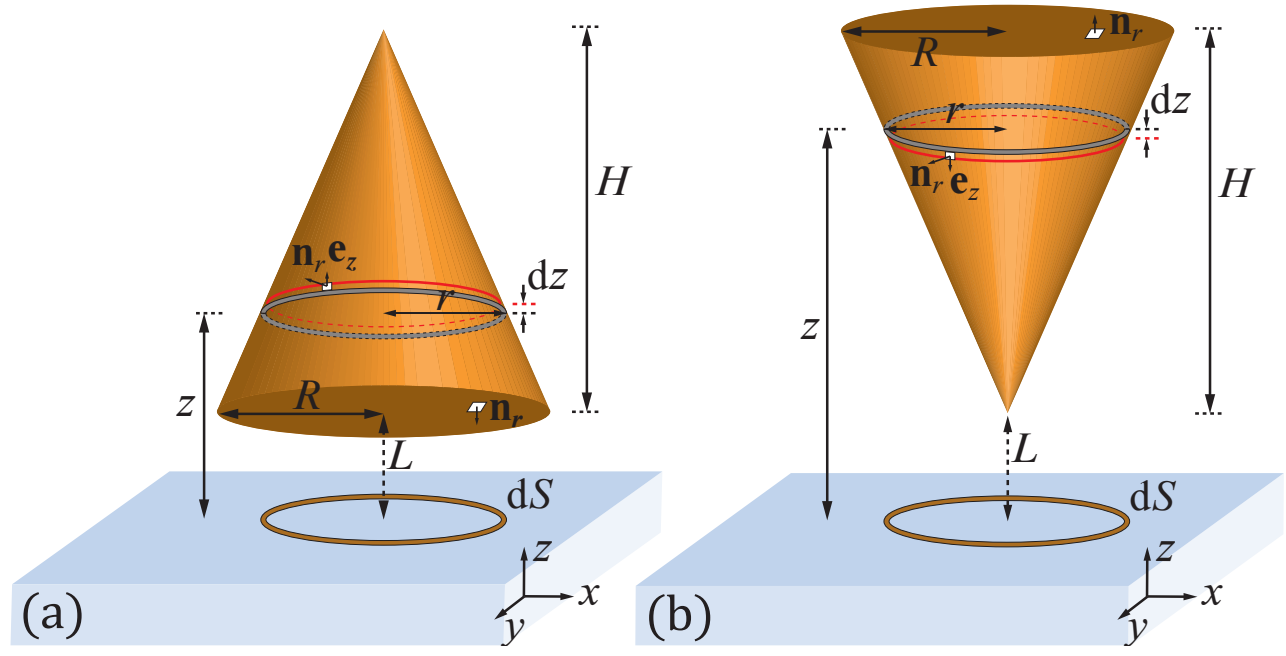

Figure 1. Geometry of the SIA for the interaction between a right circular cone and a plate, in several orientations of the conic particle. The conical shape is characterized by its base radius $R$ and height $H$. In (a) the cone's base is toward the plate, and the separation between them is $L$. The $z$-component $\mathbf{e}_{z}$ of the normal vector $\mathbf{n}_{r}$ is with a plus sign on the lateral surface, and with a minus sign on the base where $\mathbf{n}_{r} \equiv-\mathbf{e}_{z}$. If one moves along a slant height, from the cone's apex towards its base, he/she'll notice that $\mathbf{e}_{z}$ changes sign with a jump, when the edge is reached. Thus the general expression for the force has the form Eq. (11). The projection of the infinitesimal area $\mathrm{d} S$ of the lateral surface is between two concentric circumference with radiuses $r$ and $r+\mathrm{d} r$ and center lying on $H$. The considerations made is case (a) are altogether applicable for the case where the apex of the cone is toward the plate (b).

Now if one applies Eq. (9) for the case of a conical colloidal particle which base is toward the plate $(\triangleleft \mid)$ [see Fig. 1(a)], with the assumption that the interaction is induced by point-like sources, the result reads

$$
F_{\text {SIA }}^{\triangleleft \mid}(L)=-2 \pi \frac{R^{2}}{H^{2}} \int_{L}^{L+H}(L+H-z) f_{\mathscr{A}}^{\|}(z) \mathrm{d} z+\pi R^{2} f_{\mathscr{A}}^{\|}(L) .
$$

The term $(\cdots)$ in the integrand of Eq. (11) reflects how the projection to the normal to the surface of the cone in this specific orientation changes as a function of $z$. Substituting Eq. (4) [see also the text below Eq. (8)] in the above expression for the van der Waals cone-plate interaction we can write

$$
F_{\mathrm{vdW}, \mathrm{SIA}}^{\triangleleft \mid}(L)=2 \pi R^{2}(\sigma-1) H_{A}^{(\mathrm{reg})} \vartheta^{\sigma-3}\left\{\frac{1}{2 L^{\sigma}}-\frac{(L+H)^{2-\sigma}+L^{1-\sigma}[H(\sigma-2)-L]}{H^{2}(\sigma-1)(\sigma-2)}\right\} .
$$


The corresponding expression for the CCF arising between a cone and a plate for this specific geometry, following Eq. (10), can then be written as

$$
\beta F_{\mathrm{Cas}, \mathrm{SIA}}^{\triangleleft \mid}(L)=\pi R^{2} f_{\mathrm{Cas}}^{\|}(L)=\pi R^{2} L^{-3} X_{\mathrm{Cas}}^{\|}\left(x_{t}, x_{\mu}, \cdots\right) .
$$

The general expression within the SIA, for any geometry conditioned force in a system composed out of a cone which apex faces the plate $(\triangleright \mid)$ the cone interacts with [see Fig. 1(b)], has the form

$$
F_{\text {SIA }}^{\triangleright \mid}(L)=2 \pi \frac{R^{2}}{H^{2}} \int_{L}^{L+H}(z-L) f_{\mathscr{A}}^{\|}(z) \mathrm{d} z-\pi R^{2} f_{\mathscr{A}}^{\|}(L+H) .
$$

Hence for the vdWF with a free varying interaction decay parameter $\sigma$ one obtains the following expression

$$
F_{\mathrm{vdW}, \mathrm{SIA}}^{\triangleright \mid}(L)=\pi H_{A}^{(\mathrm{reg})} \vartheta^{\sigma-3} \frac{R^{2}}{H^{2}(\sigma-2)}\left[\frac{2}{L^{\sigma-2}}-\frac{2 L^{2}+2 L H \sigma+H^{2}(\sigma-1) \sigma}{(L+H)^{\sigma}}\right],
$$

whereas restricting the integration only over the surface of the cone which faces the plate, i.e., confines the fluctuations of the fluid medium, one writes for the CCF

$$
\beta F_{\text {Cas }, \text { SIA }}^{\triangleright \mid}(L)=2 \pi \frac{R^{2}}{H^{2}} \int_{L}^{L+H} \frac{z-L}{z^{3}} X_{\text {Cas }}^{\|}\left[x_{t}(z), x_{\mu}(z), \cdots\right] \mathrm{d} z \equiv 2 \pi \frac{R^{2}}{H^{2}} X_{\text {Cas }, \text { SIA }}^{\triangleright \mid}\left(H \mid x_{t}, x_{\mu}, \cdots\right) .
$$

Last we deliver the equations describing the interactions in a cone-plate system, with the consideration that the height of the cone is parallel to the plate $(\triangle \mid)$ (see Fig. 2). Therefore for the general expression and those for the vdWF and CCF one has

$$
F_{\mathrm{SIA}}^{\triangle \mid}(L)=2 H\left[\int_{L}^{L+R}\left(1-\frac{z-L}{R}\right) \mathfrak{F}_{+}(z) f_{\mathscr{A}}^{\|}(z) \mathrm{d} z-\int_{L+R}^{L+2 R}\left(\frac{z-L}{R}-1\right) \mathfrak{F}_{-}(z) f_{\mathscr{A}}^{\|}(z) \mathrm{d} z\right],
$$

where

$$
\mathfrak{\mho}_{ \pm}(z)=\ln \left[\frac{R+\sqrt{(z-L)(L+2 R-z)}}{ \pm(L+R-z)}\right] .
$$

When one considers the standard [non-retarded (London)] van der Waals interaction $\sigma=3$

$$
F_{\mathrm{vdW}, \mathrm{SIA}}^{\triangle \mid}(L \mid 3)=H_{A}^{(\mathrm{reg})} \frac{2 \pi H R^{2}}{(L+R)[L(L+2 R)]^{3 / 2}},
$$

while for the retarded (Casimir-Polder) one $\sigma=4$ and the force becomes

$$
F_{\mathrm{vdW}, \mathrm{SIA}}^{\triangle \mid}(L \mid 4)=H_{A}^{(\mathrm{reg})} \vartheta \frac{\pi H R^{2}(2 L+R)(2 L+3 R)}{(L+R)^{2}[L(L+2 R)]^{5 / 2}} .
$$

In view of Eqs. (10) and (17a) for the CCF in this specific geometry one finds

$$
\begin{aligned}
F_{\text {Cas }, \text { SIA }}^{\Delta \mid}(L) & =2 H \int_{L}^{L+R}\left(1-\frac{z-L}{R}\right) \frac{\mathfrak{F}_{+}(z)}{z^{3}} X_{\text {Cas }}^{\|}\left[x_{t}(z), x_{\mu}(z), \cdots\right] \mathrm{d} z \\
& \equiv 2 H X_{\text {Cas,SIA }}^{\triangle \mid}\left(R \mid x_{t}, x_{\mu}, \cdots\right) .
\end{aligned}
$$

If interested, the reader can refer to Appendix A.2 of [18] for the detail derivation of the infinitesimal area elements appearing in Eqs. (11), (14) and (17a). 


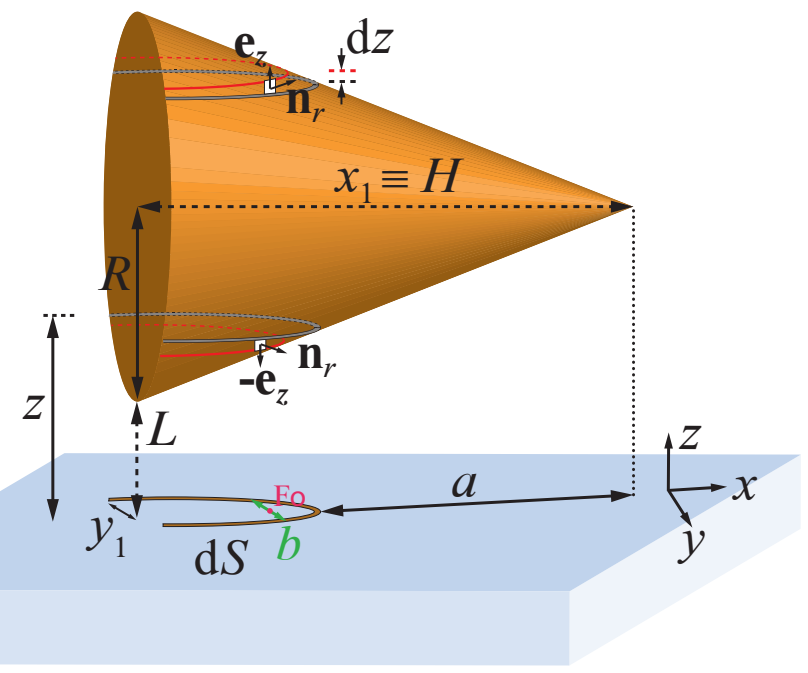

Figure 2. Geometry of the surface integration approach for the interaction between a right circular cone and a plate, when the orientation of the cone is such that $H$ is parallel to the plate. In this scenario the infinitesimal projection $\mathrm{d} S$ is the area enclosed between two hyperbolae. In order to obtain an expression for $\mathrm{d} S$, we make use of the expression of the area enclosed between a hyperbola and a line parallel to the $y$-axis (see p. 323 in [17]) $S=x_{1} y_{1}-\ln \left[\left(x_{1} / a\right)+\left(y_{1} / b\right)\right]$. Here $x_{1}$ and $y_{1}$ are the coordinates of the point of intersection between the hyperbola and the line, $a$ is the distance between the hyperbola's apex and the beginning of the coordinate frame, and $b=\sqrt{e^{2}-a^{2}}(e-$ the separation between the focus Fo of the hyperbola and the beginning of the coordinate frame).

Since SIA utilize the knowledge of the behavior of the force per unit area arising between a pair of parallel plates, in the following section we present the corresponding model within which we describe such a system. Because the included expressions have already been presented in details in [2], here only key results, as well some notations, are going to be given which will be needed in the remainder of the article.

\section{The model}

As explained in [2], we consider a lattice-gas model of a fluid confined between two planar plates, separated at a distance $L$ from each other, with grand canonical potential $\Omega[\rho(\mathbf{r})]$ given by

$$
\begin{aligned}
\Omega[\rho(\mathbf{r})] & =k_{B} T \sum_{\mathbf{r} \in \mathbb{M}}\{\rho(\mathbf{r}) \ln [\rho(\mathbf{r})]+[1-\rho(\mathbf{r})] \ln [1-\rho(\mathbf{r})]\}+\frac{1}{2} \sum_{\mathbf{r}, \mathbf{r}^{\prime} \in \mathbb{M}} \rho(\mathbf{r}) w^{l}\left(\mathbf{r}-\mathbf{r}^{\prime}\right) \rho\left(\mathbf{r}^{\prime}\right) \\
& +\sum_{\mathbf{r} \in \mathbb{M}}\left[V^{\left(s_{1}|l| s_{2}\right)}(z)-\mu\right] \rho(\mathbf{r}),
\end{aligned}
$$

where $\mathbb{M}$ is a simple cubic lattice in the region occupied by the fluid medium $-\infty^{d-1} \times[0, L]$ and $V^{\left(s_{1}|l| s_{2}\right)}(z)$ is an external potential that reflects the interactions between the confining plates and the constituents of the fluid. In Eq. (18) $w^{l}\left(\mathbf{r}-\mathbf{r}^{\prime}\right)=-4 J^{l}\left(\mathbf{r}-\mathbf{r}^{\prime}\right)$ is the nonlocal coupling (interaction potential) between the constituents of the confined medium and $\mu$ is the chemical potential. All length scales here and in the remainder are taken in units of the lattice constant $a_{0}$, so that the particle number 
density $\rho(\mathbf{r})$ becomes a number which varies in the range $[0,1]$. We recall that in the framework of a mean-field treatment with respect to the critical behavior the effective spatial dimension is $d=4$, irrespective of the actual dimension of the model under consideration.

In Eq. (18)

$$
V^{\left(s_{1}|l| s_{2}\right)}(z)=-\rho_{s_{1}} J_{\mathrm{sr}}^{s_{1}, l} \delta(z)-\rho_{s_{2}} J_{\mathrm{sr}}^{s_{2}, l} \delta(L-z)+v_{s_{1}}(z+1)^{-\sigma}+v_{s_{2}}(L+1-z)^{-\sigma},
$$

where $v_{s_{i}}=-G(d, \sigma) \rho_{s_{i}} J^{s_{i}, l}, i=1,2$, with

$$
G(d, \sigma)=4 \pi^{(d-1) / 2} \frac{\Gamma\left(\frac{1+\sigma}{2}\right)}{\sigma \Gamma\left(\frac{d+\sigma}{2}\right)},
$$

and $\delta(x)$ is the discrete delta function;

$$
J^{l}(\mathbf{r})=J_{\mathrm{sr}}^{l}\{\delta(|\mathbf{r}|)+\delta(|\mathbf{r}|-1)\}+\frac{J^{l} \theta(|\mathbf{r}|-1)}{1+|\mathbf{r}|^{d+\sigma}},
$$

is a proper lattice version of $-w^{l}(\mathbf{r}) / 4$ as the interaction energy between the fluid particles, and

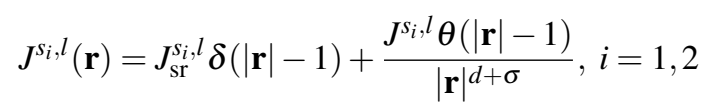

is the one between a fluid particle and a substrate particle, $\theta(x)$ is the Heaviside step function with the convention $\theta(0)=0$.

Taking into account the translational symmetry of the system along the bounding surfaces, the variation with respect to $\rho(\mathbf{r})$ leads to an equation of state for the equilibrium density $\rho^{*}(\mathbf{r})=$ $\left[1+\phi^{*}(\mathbf{r})\right] / 2$, where $\phi(\mathbf{r}) \equiv \phi\left(\mathbf{r}_{\|}, z\right)=\phi(z)$, with $\mathbf{r}=\left\{\mathbf{r}_{\|}, z\right\}$ is the local order parameter profile $\{\phi(z), 0 \leq z \leq L\}$. In terms of $\phi(z)$ the equation of state can be written in the following form

$$
\begin{aligned}
\operatorname{arctanh}\left[\phi^{*}(z)\right] & =\frac{\beta}{2}[\Delta \mu-\Delta V(z)]+K\left\{\mathrm{a}_{d, \sigma}(\lambda) \phi^{*}(z)+\mathrm{a}_{d, \sigma}^{n n}(\lambda)\left[\phi^{*}(z+1)+\phi^{*}(z-1)\right]\right. \\
& \left.+\lambda \sum_{z^{\prime}=0}^{L} g_{d, \sigma}\left(\left|z-z^{\prime}\right|\right) \theta\left(\left|z-z^{\prime}\right|-1\right) \phi^{*}\left(z^{\prime}\right)\right\}
\end{aligned}
$$

where $\Delta \mu=\mu-\mu_{c}, K=\beta J_{\mathrm{sr}}^{l}, \mathrm{a}_{d, \sigma}(\lambda)=(2 d-1)+\lambda\left(c_{d, \sigma}-d\right)$ and $\mathrm{a}_{d, \sigma}^{n n}(\lambda)=1.0+\lambda\left(c_{d, \sigma}^{n n}-0.5\right)$ with $c_{d, \sigma}^{n n}=g_{d, \sigma}(1)+g_{d, \sigma}^{n n}( \pm 1)$. The functions $c_{d, \sigma}, g_{d, \sigma}\left(\left|z-z^{\prime}\right|\right)$ and $g_{d, \sigma}^{n n}\left(\left|z-z^{\prime}\right|\right)$ are determined in Eqs. (C10), (C11) and (C12) of [19], respectively.

The excess grand canonical potential per unit area, $\omega_{\mathrm{ex}} \equiv \lim _{\mathscr{A} \rightarrow \infty}[\Omega / \mathscr{A}]-L \omega_{\text {bulk }}$, has the form

$$
\begin{aligned}
\beta \omega_{\mathrm{ex}} & =\sum_{z=0}^{L}\left\{\frac{1}{2} \ln \left[1-\phi^{*}(z)^{2}\right]-\frac{1}{2} \ln \left[1-\phi_{b}^{2}\right]+\frac{1}{4} \phi^{*}(z) \ln \left[\frac{1+\phi^{*}(z)}{1-\phi^{*}(z)}\right]-\frac{1}{4} \phi_{b} \ln \left[\frac{1+\phi_{b}}{1-\phi_{b}}\right]\right. \\
& \left.+\frac{\beta}{2} \Delta V(z) \phi^{*}(z)-\frac{\beta \Delta \mu}{2}\left[\phi^{*}(z)-\phi_{b}\right]\right\}+\beta \omega_{\text {reg }},
\end{aligned}
$$

where

$$
\beta(\sigma-1) \omega_{\mathrm{reg}}=\left[\frac{K}{K_{c}}\left(s_{1, c}+s_{2, c}\right)-\frac{1}{4} G(d, \sigma) K \lambda\right] \frac{\vartheta^{\sigma-d}}{L^{\sigma-1}}
$$


Here $\phi_{b}$ is the bulk value of the order parameter (see Eq. (4.2) in [2]), $K_{c}=\beta_{c} J_{\mathrm{sr}}^{l}$ and $s_{i, c}, i=1,2$ are the values of the plates-fluid coupling parameters evaluated at the bulk critical point of the system $\left\{\beta=\beta_{c}=\left[\sum_{\mathbf{r}} J^{l}(\mathbf{r})\right]^{-1}, \mu=\mu_{c}=-2 \sum_{\mathbf{r}} J^{l}(\mathbf{r})\right.$, with the sum running over the whole lattice $\}$.

The effective surface potential $\beta \Delta V(z) / 2$ in Eqs. (23) and (24) is given by

$$
\frac{\beta}{2} \Delta V(z)=\frac{s_{1}}{(z+1)^{\sigma}}+\frac{s_{2}}{(L+1-z)^{\sigma}}
$$

where $1 \leq z \leq L-1$ and

$$
s_{i}=\frac{1}{2} \beta G(d, \sigma)\left(\rho_{s_{i}} J^{s_{i}, l}-\rho_{c} J^{l}\right), i=1,2
$$

are the ( $T$ - and $\mu$-independent) dimensionless plates-fluid coupling parameters $\propto x_{s_{i}}$. In Eq. (26) the restriction $z \geq 1$ holds because we consider the layers closest to the substrate to be completely occupied by the liquid phase of the fluid, i.e., $\phi(0)=\phi(L)=1$, thus ensuring the $(+,+)$ boundary conditions. Physically this can be accomplished by choosing a proper coating of the surfaces of the plates. The coupling parameter $\lambda \propto x_{l}$ probes the importance of the long-ranged parts of the interaction potential within the fluid medium

$$
\lambda=J^{l} / J_{\mathrm{sr}}^{l}
$$

In Eq. (27) $s_{i}>0$, i.e., $\rho_{s_{i}} J^{s_{i}, l}>\rho_{c} J^{l}$ corresponds to plates "preferring" the "liquid" phase of the fluid, while $s_{i}<0$, or $\rho_{s_{i}} J^{s_{i}, l}<\rho_{c} J^{l}$ mirrors the one with affinity to its "gas" phase. If the interactions between the constituents of the fluid are of Lennard-Jones type one has $\lambda=2$, as commented in Refs. $[2,19]$. The marginal case $s_{i}=0$ together with $\lambda=0$ describes a pure short-range system (for details refer to Eqs. (4.10) and the text therein in [2]).

The expressions given so far provide the basis of the model that will be used to determine the cone-plate interactions in the remainder.

Using Eqs. (2) and (24) for the total force $f_{\text {tot }}^{\|}(L \mid T, \mu)$ (per unit area $\mathscr{A}$ and $k_{B} T$ ) acting between parallel plates bounding the fluid medium the following expression can be written

$$
f_{\text {tot }}^{\|}(L \mid T, \mu)=-\frac{\beta}{2}\left[\omega_{\mathrm{ex}}(L+1 \mid T, \mu)-\omega_{\mathrm{ex}}(L-1 \mid T, \mu)\right]-\frac{4 K s_{1, c} s_{2, c}}{G(d, \sigma) K_{c}^{2} \lambda} L^{-\sigma} \vartheta^{\sigma-d},
$$

where the last term represents the direct interaction between the plates (for details see the Appendix in [2]). On the other hand, if one subtracts from the potential $\omega_{\mathrm{ex}}$ its regular part $\omega_{\text {reg }}$, i.e., if we consider the quantity

$$
\Delta \omega \equiv \lim _{\mathscr{A} \rightarrow \infty}\left[\left(\Omega-\Omega_{\text {reg }}\right) / \mathscr{A}\right]-L \omega_{\text {bulk }}
$$

then, in accord with Eqs. (1) - (3), the $L$ dependence of $\Delta \omega$ via Eq. (2) provides the singular part of the total force, i.e., $f_{\mathrm{Cas}}^{\|}(L \mid T, \mu)$. Explicitly, one has

$$
f_{\mathrm{Cas}}^{\|}(L \mid T, \mu)=-\frac{\beta}{2}[\Delta \omega(L+1 \mid T, \mu)-\Delta \omega(L-1 \mid T, \mu)] .
$$

Thus, near $T_{c}$ the TF and the CCF are related via the expression

$$
f_{\mathrm{tot}}^{\|}(L \mid T, \mu)=f_{\mathrm{Cas}}^{\|}(L \mid T, \mu)+(\sigma-1) \beta H_{A}^{(\mathrm{reg})} L^{-\sigma} \vartheta^{\sigma-d},
$$

where the last term is the mathematical equivalent of $F_{\mathrm{vdW}}$ in Eq. (3) per unit area $\mathscr{A}$ and $k_{B} T$ for a film geometry with $H_{A}^{(\mathrm{reg})}$ being the nonsingular (regular) part of the Hamaker term given by

$$
(\sigma-1) \beta H_{A}^{(\mathrm{reg})}=-\frac{4 K s_{1, c} s_{2, c}}{G(d, \sigma) K_{c}^{2} \lambda}+\frac{K}{K_{c}}\left(s_{1, c}+s_{2, c}\right)-\frac{1}{4} G(d, \sigma) K \lambda .
$$


For the singular part of $H_{A}$ within the presented model the following expression was derived (see Eq. (A6) in [2])

$$
(\sigma-1) \beta H_{A}^{(\text {sing })}(T, \mu)=\frac{K}{K_{c}}\left(s_{1, c}+s_{2, c}\right) \phi_{b}-\frac{1}{4} G(d, \sigma) K \lambda \phi_{b}^{2},
$$

where the $T$ and $\mu$ dependance is carried by the bulk order parameter $\phi_{b}$ (see Eq. (4.2) in [2]).

In the next section, based on the results reported in Subsec. 2.2 and Sec. 3, we present numerical results for the behaviour of the above discussed forces in cone-plate fluid system for the cases $d=$ $\sigma=3$.

\section{Results}

In this section, using the results for $d=\sigma=4$ from the mean-field type numerical study discussed in the text above, together with the SIA approximation, we will present some results for the behavior of the CCF, vdWF and TF between a conical particle and a plate for several orientations of the colloid with respect to the smooth planar substrate in $d=3$. As it became clear from the above shown equations, the key knowledge which is required for the desired calculations to be performed, is the force [scaling function(s)] per unit area between two parallel plates, for many different separations $L$, and at various values of $T$ and $\mu$ of the fluctuating fluid medium.

Within the mean-field theory the $T$ and $\mu$ dependance of the corresponding forces near the bulk critical point $\left(T=T_{c}, \Delta \mu=0\right)$ is given by the temperature and field scaling variables $x_{t}$ and $x_{\mu}$, respectively [see the text beneath Eq. (5)], with $v=1 / 2$ and $\Delta=3 / 2$. In our numerical treatment we take these variables to range in the intervals: $x_{t} \in\left[-24^{2} ; 24^{2}\right]$ and $x_{\mu} \in\left[-24^{3} ; 24^{3}\right]$. For the study of the scaling function of the CCF, the separation $L$ between the set of parallel plates is varied from 20 to 60 with step 2, from 25 to 95 with step 5 and from 70 to 200 with step 10 . In order to demonstrate the effect of the sign change of the considered forces and in view of potential experimental realization of the predicted effects we choose the following values of the coupling parameters: $s_{1}=1.0, s_{2}=-0.01$ and $\lambda=2.0$. The physical argumentation of the so chosen values is explained in Section IV C of [1].

\subsection{Calculation of the forces in the cone-plate system within the SIA in $d=3$}

From Eq. (12), considering only genuine van der Waals interactions $(d=\sigma)$ in $d=3$, one has that within the SIA the force is calculated via the following expression

$$
L \beta F_{\mathrm{vdW}, \mathrm{SIA}}^{\triangleleft \mid}(L) / \pi \Xi_{R}^{2}=2 \beta H_{A}^{(\mathrm{reg})} \frac{\Xi_{H}}{1+\Xi_{H}},
$$

where we have introduced the notations $\Xi_{R} \equiv R / L$ and $\Xi_{H} \equiv H / L$. Accordingly for the CCF from Eq. (13) one can write

$$
L \beta F_{\text {Cas }, \text { SIA }}^{\triangleleft \mid}(L) / \pi \Xi_{R}^{2}=X_{\text {Cas }}^{\|}\left(x_{t}, x_{\mu}, \cdots\right),
$$

i.e. its behavior coincides with that of the plate-plate scaling function obtained for a desired separation $L$.

For the forces of interest in the remaining configurations one has:

(i) from Eq. (15) with $\sigma=3$ the following

$$
L \beta F_{\mathrm{vdW}, \mathrm{SIA}}^{\triangleright \mid}(L) / \pi \Xi_{R}^{2}=2 \beta H_{A}^{(\mathrm{reg})} \frac{\Xi_{H}}{\left(1+\Xi_{H}\right)^{3}} .
$$


(ii) With the use of Eq. (16), one can evaluate the scaling function entering there and hence the CCF vie

$$
\begin{aligned}
X_{\mathrm{Cas}, \mathrm{SIA}}^{\triangleright \mid} & =\int_{1}^{\Lambda_{1}} I^{\triangleright \mid}\left(\bar{z} \mid \Lambda_{1}\right) X_{\mathrm{Cas}}^{\|}\left[\mathfrak{a}_{1}(\bar{z})\right] \mathrm{d} \bar{z}+\sum_{i=j+1}^{j_{\max }-1} \int_{\Lambda_{i-1}}^{\Lambda_{i}} I^{\triangleright \mid}\left(\bar{z} \mid \Lambda_{i}\right) X_{\mathrm{Cas}}^{\|}\left[\mathfrak{a}_{i}(\bar{z})\right] \mathrm{d} \bar{z} \\
& +\int_{\Lambda_{\max }}^{1+\Xi_{H}} I^{\triangleright \mid}\left(\bar{z} \mid \Lambda_{\max }\right) X_{\mathrm{Cas}}^{\|}\left[\mathfrak{a}_{\max }(\bar{z})\right] \mathrm{d} \bar{z}, \quad \text { with } L \beta F_{\mathrm{Cas}, \mathrm{SIA}}^{\triangleright \mid} / \pi \Xi_{R}^{2}=\frac{2}{\Xi_{H}^{2}} X_{\text {Cas,SIA }}^{\triangleright \mid}
\end{aligned}
$$

where

$$
I^{\triangleright \mid}\left(\bar{z} \mid \Lambda_{j}\right)=\frac{\bar{z}-1}{\bar{z}^{3}} \theta\left(\Xi_{H}-\Lambda_{j}\right),
$$

with $\Lambda_{j} \equiv L_{j} / L, j=1, j_{\max } ; \bar{z} \equiv z / L$ is dimensionless variable; $L_{\max } \equiv L_{j_{\max }}$ is the largest system for which numerical data are available, the arguments of the scaling function $\left[\mathfrak{a}_{j}(\bar{z})\right] \triangleq$ $\left[x_{t}\left(L_{z ; j}\right), x_{\mu}\left(L_{z ; j}\right), \ldots\right], L_{z ; j} \equiv \bar{z} L_{j}$ and $\theta$ is the Heaviside step function with the convention $\theta(0)=0$. In the calculations performed in the current article $L_{\max }=200$.

(iii) Last we give the expressions used to evaluate the vdWF and the CCF for the case when the height of the cone is parallel to the plate. Indeed, performing the dimensionalization with respect to $L$ in Eq. (17c) we have

$$
L \beta F_{\mathrm{vdW}, \mathrm{SIA}}^{\triangle \mid}(L) / \pi \Xi_{R}^{2}=2 \beta H_{A}^{(\mathrm{reg})} \frac{\Xi_{H}}{\left(1+2 \Xi_{R}\right)^{3 / 2}\left(1+\Xi_{R}\right)} .
$$

(iv) For the scaling function of the CCF from Eq. (17e), one has

$$
\begin{aligned}
X_{\mathrm{Cas}, \mathrm{SIA}}^{\triangle \mathrm{I}} & =\int_{1}^{\Lambda_{1}} I^{\triangle \mathrm{I}}\left(\bar{z} \mid \Lambda_{1}\right) X_{\mathrm{Cas}}^{\|}\left[\mathfrak{a}_{1}(\bar{z})\right] \mathrm{d} \bar{z}+\sum_{i=j+1}^{j_{\max }-1} \int_{\Lambda_{i-1}}^{\Lambda_{i}} I^{\triangle \mathrm{I}}\left(\bar{z} \mid \Lambda_{i}\right) X_{\mathrm{Cas}}^{\|}\left[\mathfrak{a}_{i}(\bar{z})\right] \mathrm{d} \bar{z} \\
& +\int_{\Lambda_{\max }}^{1+\Xi_{R}} I^{\triangle \mid}\left(\bar{z} \mid \Lambda_{\max }\right) X_{\mathrm{Cas}}^{\|}\left[\mathfrak{a}_{\max }(\bar{z})\right] \mathrm{d} \bar{z}, \text { with } L \beta F_{\mathrm{Cas}, \mathrm{SIA}}^{\triangle \mid} / \pi \Xi_{R}^{2}=\frac{2 \Xi_{H}}{\pi \Xi_{R}^{2}} X_{\mathrm{Cas}, \mathrm{SIA}}^{\triangle \mid}
\end{aligned}
$$

where

$$
I^{\triangle \mid}\left(\bar{z} \mid \Lambda_{j}\right)=\left(1-\frac{\bar{z}-1}{\Xi_{R}}\right) \frac{\mathfrak{F}_{+}(\bar{z})}{\bar{z}^{3}} \theta\left(\Xi_{R}-\Lambda_{j}\right) .
$$

In the above expressions the critical component $X_{\text {crit }}^{\|}$of the scaling function $X_{\text {Cas }}^{\|}$calculated within the mean-field theory is normalized by the procedure described in-and-out in Section V of [2], so that it contributes properly to the CCF and hence to the total force of interaction when $d<4$. The need of such normalization is explained in details in [19] (see there Sections IV.A.1 and IV.A.3).

After presenting the mathematical means to calculate the CCF and vdWF, we now pass to the detailed discussion of the results and argumentation of the experimental feasibility of the parameters used in the model calculations utilizing the SIA.

\subsection{Discussion and concluding remarks}

In the present section for physical reasons related to future experimental studies we will assume that the conical particle has either ruthenium $(\mathrm{Ru})$ or platinum $(\mathrm{Pt})$ core, while the planar substrate is made either of carbon $(\mathrm{C})$ or silica $\left(\mathrm{SiO}_{2}\right)$ aerogels. The contact surface of any of the interacting objects is considered coated by monolayer of lead $(\mathrm{Pb})$ or thallium nitride $(\mathrm{TIN})$ to ensure the $(+,+)$ boundary conditions. Finally, we complete the so assembled system by adopting xenon (Xe) as the 

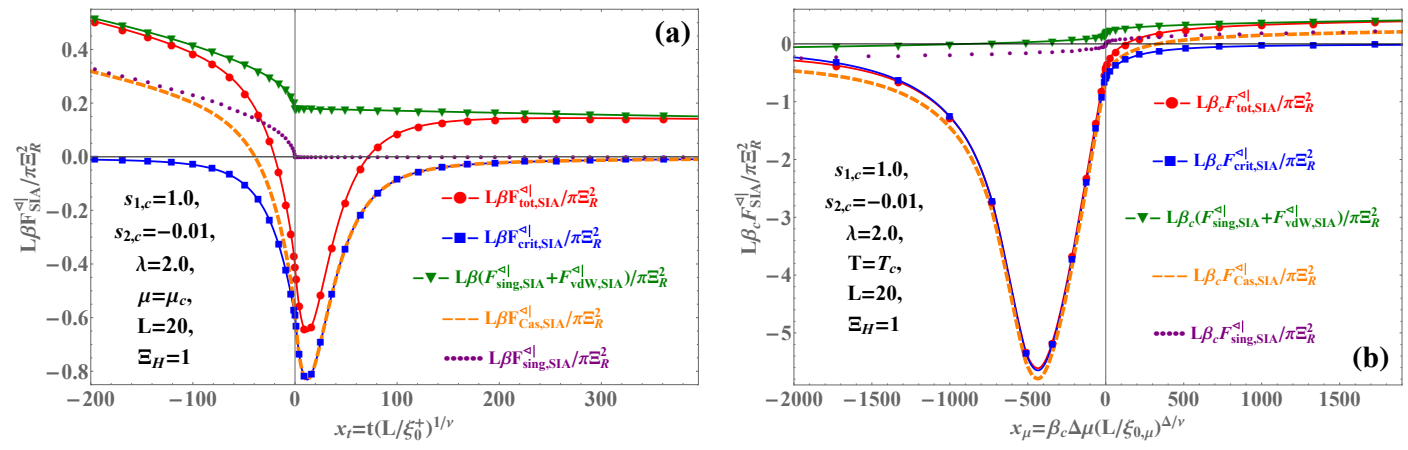

Figure 3. Behaviour of the discussed forces (multiplied by the separation $L$ and normalized by $k_{B} T$ and the area of the cones's base $\pi \Xi_{R}^{2}$ ) in $d=3$ for the case where the base of the cone is facing the plate the cone interacts with. On both figures the interplay between the critical $F_{\text {crit,SIA }}^{\triangleleft \mid}(-\square)$ and singular $F_{\text {sing,SIA }}^{\triangleleft \mid}(\bullet \bullet)$ components of the net Casimir force $F_{\text {Cas,SIA }}^{\triangleleft \mid}(---)$ is shown, as well as that between $F_{\text {Cas,SIA }}^{\triangleleft \mid}$ and the van der Waals interaction $F_{\text {vdW,SIA }}^{\triangleleft \mid}(\rightarrow)$ resulting in the total force $F_{\text {tot,SIA }}^{\triangleleft \mid}(-\bullet)$. On $(\mathbf{a})$ the temperature dependance at $\Delta \mu=0.0,\left(x_{\mu}=0.0\right)$ is depicted, while on $(\mathbf{b})$ that of the field one at $T=T_{c},\left(x_{t}=0.0\right)$. The minimal separation between the objects confining the fluid medium is taken $L=20$, where the cones's normalized height is taken $\Xi_{H}=1$ and the parameters characterizing the interactions in the systems have the following values: $\lambda=2.0$, $s_{2, c}=-0.01$ and $s_{1, c}=1.0$.

critical fluctuating medium in which the interacting objects are immersed. For detail comment and argumentation on the choice of the presented substances the reader can refer to [1].

We open the discussion with the results presented on Fig. 3. Here the interplay between all of the aforementioned forces is clearly depicted. For the chosen separation $L=20 a_{0} \simeq 12 \mathrm{~nm}$ and substances, characterized by the set of coupling parameters $\left\{s_{1, c} ; s_{2, c} ; \lambda\right\}, F_{\text {crit,SIA }}^{\triangleleft \mid}$ is negative for any value of $x_{t}$ and $x_{\mu}$, i.e., it corresponds to attraction between the cone and the plate in the entire temperature and filed intervals. Here $a_{0}$ is the distance between the xenon atoms at the critical point. From the data used here one can estimate that at $T=1.0058 T_{c}$ this component of the Casimir force reaches its minimum, with a value of $-0.9 \Xi_{R}^{2} \mathrm{pN}$. With regard only to Fig. $3(\mathbf{a})$, at $\Delta \mu=0$ for $T>T_{c}$ one has $\phi_{b}=0$ and hence, substituting Eq. (34) instead of $H_{A}^{(\text {reg })}$ into Eq. (35) we see that $F_{\text {sing,SIA }}^{\triangleleft \mid}$ is zero. On the other hand for $T<T_{c}$ the bulk order parameter $\phi_{b} \neq 0$ as the singular part of the Hamaker term (and consequently $F_{\text {sing,SIA }}^{\triangleleft \mid}$ ) corresponds to repulsion. Therefore, the behavior of net Casimir interaction $F_{\text {Cas,SIA }}^{\triangleleft \mid}=F_{\text {sing.SIA }}^{\triangleleft \mid}+F_{\text {crit,SIA }}^{\triangleleft \mid}$ coincides with that of its critical component above $T_{c}$, but one observes a single sign change at $T=0.9805 T_{c}$ in the "liquid" phase of the fluid. The superposition between the $\operatorname{vdWF} F_{\mathrm{vdW}, \text { SIA }}^{\triangleleft \mid}$ and $F_{\text {sing,SIA }}^{\triangleleft \mid}$, leads to interaction which is positive for any temperature and shows explicit decrease with the increase of the temperature like $T^{-1}$ [see Eqs. (33) and (34)]. As a result the total force $F_{\text {tot,SIA }}^{\triangleleft \mid}$ becomes repulsive outside the critical region, changes sign twice at $T_{1}=0.9901 T_{c}$ and $T_{2}=1.036 T_{c}$ after which turns attractive only near the critical point. It reaches minimum for $T_{\min }=1.0061 T_{c}$, which magnitude is $-0.71 \Xi_{R}^{2} \mathrm{pN}$. The nature of this observation is dictated by the increased correlations between the fluctuations in the fluid close to $T_{c}$. 

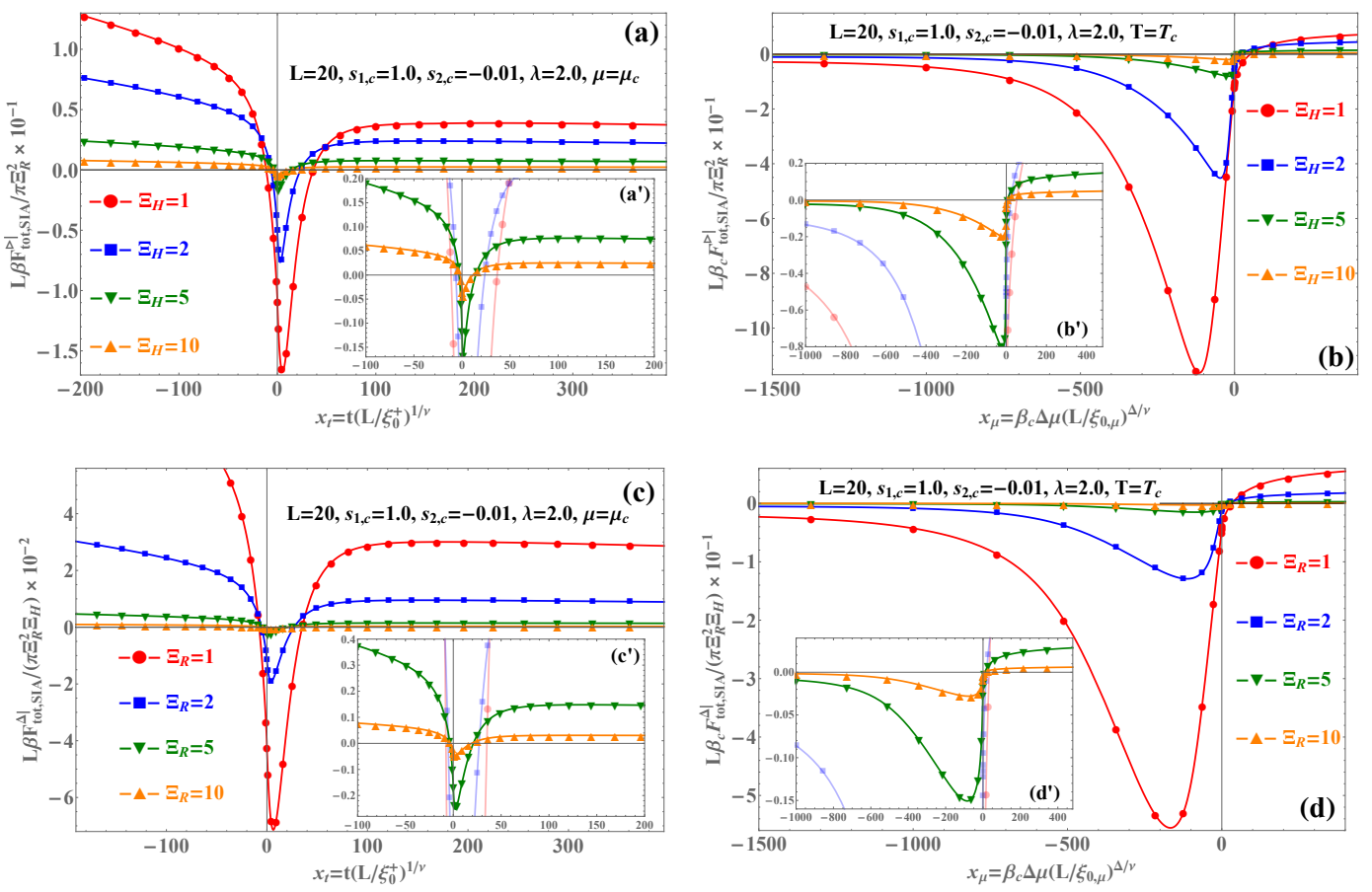

Figure 4. Behavior of the total force $F_{\text {tot,SIA }}$ (multiplied by the separation $L$ and normalized by $k_{B} T$ and the area of the cones's base $\left.\pi \Xi_{R}^{2}\right)$ in $d=3$ for the cases where: [ $(\mathbf{a})$ and $\left.(\mathbf{b})\right]$ the cone's apex is toward the plate and [(c) and (d)] the height of the cone is parallel to the plate the cone interacts with. On (a) and (c) the temperature dependance at $\Delta \mu=0.0,\left(x_{\mu}=0.0\right)$ is depicted, while on (b) and (d) that of the field one at $T=T_{c},\left(x_{t}=0.0\right)$. The upper two subfigures illustrate how $F_{\text {tot,SIA }}^{\triangleright \mid}$ changes with the increase of the cone's height $\Xi_{H}$, and on the lower ones how $F_{\text {tot,SIA }}^{\Delta \mid}$ is influenced by the variation of the base radius $\Xi_{R}$. There the force is additionally normalized by $\Xi_{H}$. The minimal separation between the objects confining the fluid medium is taken $L=20$ and the parameters characterizing the interactions in the systems have the following values: $\lambda=2.0, s_{2, c}=-0.01$ and $s_{1, c}=1.0$.

The typical behavior of $F_{\text {sing,SIA }}^{\triangleleft \mid}$ as a function of the chemical potential at $T=T_{c}$ is presented on Fig. 3(b). The force is attractive in the "gas" phase of the fluid, i.e., for $x_{\mu}<0$ and repulsive otherwise with an infinite slope at the critical point, where a sign change occurs. Hence, the CCF changes sign once in the "liquid" phase $\left(x_{\mu}>0\right)$ at $\beta_{c} \Delta \mu \simeq 1.2495 \times 10^{-3}$ and its minimum increases in magnitude slightly in comparison to $F_{\text {crit,SIA }}^{\triangleleft \mid}$, as $\left.\left(F_{\text {Cas,SIA }}^{\triangleleft \mid} / F_{\text {crit,SIA }}^{\triangleleft \mid}\right)\right|_{\text {min }}=1.0253$, where $F_{\text {crit,SIA }}^{\triangleleft \mid}\left(x_{\mu, \min }\right)=-6.1077 \Xi_{R}^{2}$ pN. From Eq. (33) follows that the vdWF does not depend on $\mu$, as a result of which the singular component of the CCF only "shifts up" and changes sign for $\mu<\mu_{c}$ at $\beta_{c} \Delta \mu \simeq-3.293 \times 10^{-3}$. When add up to the Casimir force component stemming from the finite-size contributions, the total force exhibits behavior similar to that of $F_{\text {crit,SIA }}^{\triangleleft 1}$, but now the sign change occurs closer to the critical point at $\beta_{c} \Delta \mu \simeq 0.4794 \times 10^{-3}$.

Some results about the behavior of the total force in a cone-plate system with geometry $(\triangleright \mid)$ are presented on Fig. 4(a) and (b). The first obvious result we notice is that for fixed radius of the base, 
the force decreases with the increase of the height like $\Xi_{H}^{-2}$ [see Eqs. (37a) and (37b)]. This is easily understood given that the CCF strongly depends on the confining area of the object's surface. When $\Xi_{H}$ is increased at fixed $\Xi_{R}$ the area of the surface elements, which are close to the plate and hence have higher contribution weight, decreases which in turn results in critical Casimir force with lower strength. The same is in a way true for the vdWF, but in this case the decrease is caused from the decline of the number of constituents in the volume near the cone's apex which are near the surface of the plate. As a function of $x_{t}$ the force changes sign twice, as the temperature for which it occurs in the "gas" phase decreases towards $T_{c}$ with the increase of $\Xi_{H}$, and viceversa for $T>T_{c}$. A simple quantitative assessment indicates that $t_{1}=-4.633 \times 10^{-3}$ and $t_{2}=12.776 \times 10^{-3}$, where $t_{i} \equiv\left[T_{i}\left(\Xi_{H}=\right.\right.$ $\left.1)-T_{i}\left(\Xi_{H}=10\right)\right] / T_{i}\left(\Xi_{H}=10\right), i=1,2$ with $T_{1}\left(\Xi_{H}=1\right)=0.9944 T_{c}$ and $T_{2}\left(\Xi_{H}=1\right)=1.0183 T_{c}$. The temperature at which the minimum of the force is expected to occur also decreases towards $T_{c}$ when $\Xi_{H}$ is increased. In addition, for the difference in the magnitude of the force at the point where it reaches its minimum for the lowest and highest values of $\Xi_{H}$ considered here we found that $\left[F_{\text {tot,SIA }}^{\triangleright \mid}\left(\Xi_{H}=1\right) / F_{\text {tot,SIA }}^{\triangleright \mid}\left(\Xi_{H}=10\right)\right]=36.1428$, where $F_{\text {tot,SIA }}^{\triangleright \mid}\left(\Xi_{H}=10\right)=-5.0155 \Xi_{R}^{2} \mathrm{fN}$.

For the same geometry, but as a function of $x_{\mu}, F_{\text {tot.SIA }}^{\triangleright \mid}$ changes sign only once for $\Delta \mu>0$, as the value of the chemical potential for which this is observed decreases towards $\mu_{c}$ with the increase of $\Xi_{H}$. The strength of the force at its minimum also decreases as does the value of $x_{\mu}$ at which it emerges. This case is quantitatively describes as follows: $\left.x_{\mu}\left(\Xi_{H}=1\right)\right|_{F_{\mathrm{tot}}=0}=52.763, x_{\mu}\left(\Xi_{H}=\right.$ $10)\left.\right|_{F_{\mathrm{tot}}=0}=4.096,\left.x_{\mu}\left(\Xi_{H}=1\right)\right|_{F_{\mathrm{tot} \text {, min }}}=-117.649,\left.x_{\mu}\left(\Xi_{H}=10\right)\right|_{F_{\mathrm{tot} \text {,min }}}=-19.683,\left[F_{\mathrm{tot}, \text { SIA }}^{\triangleright \mid}\left(\Xi_{H}=\right.\right.$ $\left.1) / F_{\text {tot,SIA }}^{\triangleright \mid}\left(\Xi_{H}=10\right)\right]_{\text {min }}=58.905$, with $F_{\text {tot,SIA }}^{\triangleright \mid}\left(\Xi_{H}=10\right)=-21.346 \Xi_{R}^{2} \mathrm{fN}$.

As far as the geometry $(\triangle \mid)$ is concerned, here we see that its differences with the one designated by $(\triangleright \mid)$ are only quantitative, and hence no special comments are needed. The results are depicted on Fig. 4(c) and (d).

For the sake of completeness, we give the critical temperature of xenon $T_{c}=289.765 \mathrm{~K}$ and the value of the critical chemical potential per $k_{B} T_{c}: \beta_{c} \mu_{c}=-16.213$, calculated using Eqs. (2.2b), (5.37b) and (8.8) from [20].

Although the magnitude of the TF together with the CCF may seems rather negligible, a comparison with say the weight of a single particle proofs otherwise. For instance, the weight of a platinum conical in shape particle of height $\Xi_{H}=100$ and base radius $\Xi_{R}=10$ is approximately $F_{\mathrm{w}}^{\mathrm{cone}} \approx 1.2 \times 10^{-2} \mathrm{pN}$. In the liquid phase of xenon at $T=206.4 \mathrm{~K}$ and $\Delta \mu=0$ one finds that $F_{\text {tot }}^{\triangleleft \mid} \approx 120 \mathrm{pN}, F_{\text {tot }}^{\triangleright \mid} \approx 1.2 \times 10^{-2} \mathrm{pN}$ and $F_{\text {tot }}^{\Delta \mid} \approx 17 \mathrm{pN}$. Therefore the repulsive part of the total force in one such concrete system is indeed capable of levitating a single particle or a system of such. Thus, one can indeed make use of the interplay between the quantum and thermodynamical fluctuations for governing the behavior of objects, say colloidal particles, at small distances. It can also provide a strategy for solving problems with handling, feeding, trapping and fixing of micro parts in nanotechnology resolving the issues related to sticking of the particles on the surface of the mechanical manipulator utilizing, e.g., the reversible dependence on the forces under minute changes of the temperature of the critical medium. One can perform grabbing of particles for small values of $x_{t}$, where the force is attractive and release them at a given spacial position after slightly increasing or decreasing of temperature achieving in that way a value of $x_{t}$ with a repulsive TF.

\section{Acknowledgements}

The author gratefully acknowledges the financial support via Contract No. DN 02/8 of Bulgarian NSF. 


\section{References}

[1] G. Valchev, D. Dantchev, Phys. Rev. E 96, 022107 (2017)

[2] G. Valchev, D. Dantchev, Phys. Rev. E 92, 012119 (2015)

[3] M.E. Fisher, P.G. de Gennes, C. R. Seances Acad. Sci. Paris Ser. B 287, 207 (1978)

[4] M. Krech, Casimir Effect in Critical Systems (World Scientific, Singapore, 1994)

[5] J.G. Brankov, D.M. Dantchev, N.S. Tonchev, The Theory of Critical Phenomena in Finite-Size Systems - Scaling and Quantum Effects (World Scientific, Singapore, 2000)

[6] H.B. Casimir, Proc. K. Ned. Akad. Wet. 51, 793 (1948)

[7] H.B.G. Casimir, D. Polder, Phys. Rev. 73, 360 (1948)

[8] H.B.G. Casimir, Physica 19, 846 (1953)

[9] E. Buks, M.L. Roukes, Europhys. Lett. 54, 220 (2001)

[10] E. Buks, M.L. Roukes, Phys. Rev. B 63, 033402 (2001)

[11] H.B. Chan, V.A. Aksyuk, R.N. Kleiman, D.J. Bishop, F. Capasso, Phys. Rev. Lett. 87, 211801 (2001)

[12] D. Dantchev, G. Valchev, J. Coll. Interf. Sci. 372, 148 (2012)

[13] R. Garcia, M.H.W. Chan, Phys. Rev. Lett. 83, 1187 (1999)

[14] V.A. Parsegian, Van der Waals Forces (Cambridge University Press, 2006)

[15] J.N. Israelachvili, Intermolecular and surface forces (Academic, London, 2011)

[16] B. Derjaguin, Kolloid Z. 69, 155 (1934)

[17] H.J. Bartsch, Mathematische Formeln (Fachbuchverlag Leipzig, 1991)

[18] G. Valchev, Ph.D. thesis (2015)

[19] D. Dantchev, F. Schlesener, S. Dietrich, Phys. Rev. E 76, 011121 (2007)

[20] D.C. Johnston, Advances in Thermodynamics of the van der Waals Fluid (Morgan \& Claypool Publishers, 2014) 